\title{
EDITORIAL
}

\section{Assessment for Learning}

\author{
Shahid Hassan \\ Department of Medical Education/Otorhinolaryngology, School of Medical Sciences, Universiti Sains \\ Malaysia
}

Recently I received an invitation from the Editor EiMJ to express my views on assessment in wide perspectives of learning as indicated in title. The notion of assessment for learning raises a number of questions! Is the assessment currently practiced is isolated from learning in a medical curriculum? Or more evidently, is the assessment in medical education is not practiced for learning so far. Seem like not really in every curriculum in medical education. What are the concerns shown by students about assessment in a curriculum? For them assessment is a nightmare and is seen as a hindrance in a joyful learning. Whatever the outcome of assessment in learning may be it is clear that despite of a number of steps taken to reduce the biases in assessment in terms of selecting the highly reliable and valid tools in summative examinations, the judgment for its logical decision is still in question. How and who will provide an answer to these questions? Should we do away with assessment altogether to make the learning a pleasure experience for students or replace the summative assessment by formative assessment to add on to an existing teaching load of supervisors? Whatever the decision is, at least we began to think of assessment for learning and that is a great thinking. Let's see how we can achieve this role of assessment with satisfaction for future learning program in medical education.
The current pitfall of assessment apart from its validity and reliability is the standard setting strategy that calls for an appropriate and logical decision in summative assessment [1]. Too much reliance on quantitative versus qualitative assessment, external versus internal examiners, summative versus formative assessment and single versus multiple instruments for decision making has not only added to the problem but it also speaks of a common issue seen in all those problems mentioned above. Identifying to address this common issue may perhaps bring assessment in line with learning to obviate the students' perception of assessment, a nightmare. The common issue that makes assessment an hindrance in real learning at least from student's perception is the decision taken on pass or fail, which often is based on performance of a single tool such as a single long case. This often denies the overall performance seen as integrated decision of multiple measures employed or in so many words it lacks triangulation of summative assessment with formative assessment [2] in a standard setting strategy. However, we must also ensure that decision of awarding a practicing license in medicine should not be at the cost of competence of a doctor as expected. Medical education is the profession that demands highly competent learning, which is measured by an authentic assessment. Now the central issue arising 
from all those problems mentioned above suggests that the current practice of assessment is not compatible with learning as indicated in notion of assessment for learning. The reason for this incompatibility of assessment and learning is that training in medical education is though authentic (real patients at workplace) the assessment is not equally authentic (not in a workplace). Most of the assessment tools in practice test the competence in a mock situation and performance of a candidate is therefore hardly measured. A workplace-based assessment (WPBA) is the need of time, which also takes into account the assessment of attitude, interpersonal skills and professionalism [3]. However, to implement WPBA in learning requires faculty development on assessment of performance (does of Miller's pyramid). We need to develop understanding of assessment as a program in order to improve knowledge about every accessible measure to face the challenges of its authenticity, reliability and validity that disregard assessment as part of learning.

How the workplace-based assessment will address all those issues to maintain the principles of good learning is interesting? Measures employed in WPBA relies more on formative than summative assessment and the assessment is carried out round the years with different situations, multiple cases, multiple examiners, and varying periods of examination [4]. Decision-making is relied upon formative assessment report compiled as the reflection of student's performance round the year and at various level of training (like in semester examination) in which internal assessors play the major role while the external assessors endorses decision of internal assessors. External assessor is not the right person to decide on a student's performance with one or two questions answered in an unlikely manner. An external examiner cannot judge 4-5 years of comprehensive training in 20-30 minutes of exposure. Internal assessors are the right person to guide external assessor in such a situation. Solution to question of quantitative over qualitative assessment can also be met with satisfaction since the decision taken on pass or fail will be integrated and triangulated sum of a student's accumulative performance rather than the performance on an individual instrument used to make the decision. Single versus multiple instruments deciding on student's fate will also be answered in WPBA since the measurements tools will not only be multiple but will also be varying in type, situation and complexity of cases used according to the stage of a student's training [5]. Student's perceptions of once a year summative assessment seen as a nightmare is not only obviating the learning a pleasure experience but is also precluding students from becoming a self-directed and collaborative learner who believe in learning, which is contextualized and experiential [6] with assessment as part and parcel of learning process. Summative examination in workplace-based assessment in a changing pattern of assessment (for learning) may still have a role but in a reverse order with formative assessment that we currently practice.

However, to bring such a drastic change in the current culture of assessment, we need to learn about skills of assessment as much as we need to learn about ingredients of good teaching to face the challenges. In educational science, assessment is linked to learning and its objectives. We need to decide on object of assessment (the what) and then measure it by means of a most reliable and valid instrument available (the how). The value that we assign to our summative assessment should reliably and validly evaluate the performance of our 
trainees. But how often is this achieved? Assessment of intellectual process (know) to clinical skills (show how) and attitude (professionalism) should ultimately find the fit between object of assessment and the measurement tools that are accessible, feasible and practical to assess the performance [7]. And this clearly points to switching over to workplace-based assessment with its evident-based models. Other two reasons to move to a new culture of workplace-based assessment are: 1) our claim of practicing the integrated (triangulation of measures within the same component of knowledge or skills domain) assessment. 2) Our satisfaction of meeting the outcome objectives of pre-set level of performance and not competency alone. Integration or triangulation is definitely not practiced in current assessment since decision taken is often based on result of an isolated instrument for example a single long case in summative assessment in most of the undergraduate and postgraduate examinations. The objectives of achieving competence in current assessment is also not met due to its inability to attain the required level of authenticity with increasing role of performance as shown in Miller's pyramid of competent learning. Mini-Cex (Mini clinical evaluation exercise) and DOPS (Direct observed procedural skills) as WPBA are considered reliable, valid and feasible instruments for assessment of clinical performance simply because, workplace based assessment is an "assessment of what doctors actually do in practice" [8] and all the postgraduate as well as undergraduate medical education programs should start practicing these as formative assessment before adapting them to replace the traditional tools in summative assessment. Workplace-based assessment is perceived as a different ball game by the students and is no scarier like traditional summative assessment.
Author experienced student's fondness for WPBA and the reason of students liking for Mini-Cex (9) and DOPS (10) encounters is due to the provision of feedback as a mandatory feature of these assessment methods, which makes it a perfect model for learning in assessment.

The concepts of integration and triangulation in assessment suggest that the decision on pass or fail should base on satisfactory or unsatisfactory performance of students both on quantitative scoring as well as on qualitative observation. A student's bad day in one assessment tool should not decide his/her fate of 4-5 years of intensive learning in medical education. This can be avoided if the mathematical total of quantitative assessment is combined with qualitatively observation within a test component (integration) or outside a test component (triangulation) of measurement tools. This is an important issue, which is grossly overlooked in our assessment of professional competencies in medical education. Integration refers to calling upon for a right qualitative judgment utilizing the complementing role of assessment tools in the same component (for example MCQ and essay questions in written and long and short cases in clinical components). This is to provide the benefit of doubt to students if the quantitative judgment of an individual instrument is in question. Triangulation refers to making a qualitative judgment based on best-practice evidences on assessment gathered over different time, under different circumstances, by different evaluators and using different methods, which is a perfect example of a workplace-based assessment tool. For example utilizing the Mini-Cex and DOPS compiled results as formative assessment to judge summative result of a borderline student. Van der Vleuten has therefore described the assessment as 
instructional design problem than measurement problem [11]. Both of these principles of assessment in medical education address the evaluation of complex competencies, which is integral part of training in postgraduate medical education and to some extent in later part of undergraduate medical education. A comprehensive judgment thus requires qualitative as well as quantitative information from different sources to decide on professional performance of an individual as safe or unsafe for future medical practice. This is amicably well addressed in workplace-based formative assessment.

In order to achieve the competency in medical education, author strongly believe that the quality of assessment needs integrated assessment program rather than the performance in individual instruments and this requires good coordination and planning. Assessment often compromises authentic judgment and a single method to be good or bad depends on the context for what it is designed to assess. A mere reliance on pass/fail as an outcome of a summative assessment based on performance of an individual instrument is against the norms of an integrated and well-triangulated assessment model. Formative assessment based on principles of evaluation of acquired competency should be practiced to satisfy a candidate achieving the quality training before allowing him to sit a so-called summative assessment in a medical education program. For correct judgment in difficult borderline cases a pre-recorded assessment result of performance at workplace carried out as formative assessment can be triangulated with highly subjective clinical component of summative assessment of trainees to decide on satisfactory versus unsatisfactory outcome. However, this overarching assessment strategy needs conjoint faculty development in workplace assessment, its rigorous implementation and record readily available for reviewing and triangulating with summative assessment results. This will be a remarkable change in present culture of assessment, which gradually will pave the way to rely on assessment of performance at workplace with high level of confidence in judgment.

In conclusion the design of assessment methods in a curriculum should not only be a perfect fit between objects of assessment and the measurement tools to accommodate the Bloom's taxonomy of knowledge and Miller's pyramid of clinical competency but it should also encourage good learning. Considering to switchover from the current culture of summative assessment to a comprehensive model of formative assessment it is important for a strategically designed assessment program to promote learning to achieve its set educational outcome. Practicing triangulation in assessment will produce reproducible result with high level of confidence. A good assessment model with this concept of learning is achievable only if we initiate the assessment of performance and professional behavior as WPBA. This help to monitor the outcome objectives of an educational program. Mini-Cex (Mini-clinical exercise), cb (Case-based) Discussion, DOPS (Direct observed procedural skills) and MSF (Multi-source feedback) of workplace-based assessment are good models to start with a formative assessment program readily available for triangulation of summative assessment. Methodical use of workplacebased formative assessment can play its due role in decision-making to achieve a logically appropriate and valid decision on pass or fail of student's performance both in undergraduate as well as in postgraduate medical education. 


\section{References}

1. Mitchell D. C. Multiple measures and high stakes decisions: A framework for combining measures. Educational Measurement Issues and Practice, 2003; 22, 2.

2. Neil Jackson, Alex Jamieson and Anwar Khan. Assessment in medical education and training. Oxford: Radcliffe Publishing, 2007.

3. John JN, Linda LB, Daniel D, Gregory SF. The Mini-CEX: a method for assessing clinical skills. Ann Intern Med. 2003; 138:476-481

4. Norcini JJ, Blank LL, Arnold GK, Kimball HR. The Mini-CEX (clinical evaluation exercise): a preliminary investigation. Ann Intern Med. 1995; 123:795-799

5. Norcini JJ, Blank LL, Arnold GK, Kimball HR. Examiner differences in the Mini-CEX. Adv Health Sci Educ. 1997; 1:27-33. http://dx.doi.org/10.1023/A:100973472365

6. Hassan S. Assessment of postgraduate Program in Otolaryngology and Head-Neck
Surgery in Malaysia - Are we Adequate. MSOHNS News Bulletin, Vol. 4, Issue 1, May 2011.

7. Hassan S. Postgraduate Assessment in Malaysia: Rationale of Decision Making. Education in Medicine Journal, 2011; 3 (1): e15. http://dx.doi.org/10.5959/eimj.3.1.2011.e1

8. Swanwick T, Chana N. Workplace-based assessment. $\mathrm{Br} J$ Hosp Med (Lond) 2009; 70:290-3.

9. Hassan S. Faculty development: Mini-Cex as workplace-based assessment. Education in Medicine Journal, 2011; 3 (1): e20-31. http://dx.doi.org/10.5959/eimj.3.1.2011.or2

10. Faculty development: DOPS as workplacebased assessment. Education in Medicine Journal, 2011; 3 (1): e32-43. http://dx.doi.org/10.5959/eimj.3.1.2011.or4

11. Van der Vleuten CPM. The assessment of professional competence: development, research and practical implications. Adv Health Sci Educ 1996; 1:41-67. http://dx.doi.org/10.1007/BF00596229

\section{Corresponding author}

Professor Dr Shahid Hassan, Medical Education/Otorhinolaryngology Department, School of Medical Sciences, Universiti Sains Malaysia, Kubang Kerian, 16150 Kota Bharu, Kelantan, Malaysia Email: shahid@kb.usm.my, gorshahi@yahoo.com 